\title{
Localization of Wideband Signals Using Least-Squares and Total Least-Squares Approaches
}

\author{
Shahrokh Valaee, Benoit Champagne, Member, IEEE, and Peter Kabal, Member, IEEE
}

\begin{abstract}
In this paper, we introduce a new focusing technique for localization of wideband signals. Relaxing the unitary assumption for the focusing matrices, we formulate the least-square (LS) and the total least-square (TLS) coherent signal-subspace methods. The TLS is an alternative to the conventional LS and uses the fact that the errors can exist both in the focusing location matrix as well as in the estimated location matrix at a given frequency bin. To prevent the focusing loss, we use a class of focusing matrices that are constant under multiplication by their Hermitian transpose. The class of unitary matrices comports with this property. We then develop a new focusing technique based on a modification to the TLS (MTLS). It is shown that the computational complexity of the new technique is significantly lower than that for the rotational signal-subspace method (RSS). The focusing gain of the new technique is also larger than the focusing gain of the RSS algorithm. The simulation study shows that, compared with the RSS, the new algorithm has a smaller resolution signal-to-noise ratio (SNR).
\end{abstract}

\section{INTRODUCTION}

W IDEBAND array processing arises in many applications such as passive sonar, microphone array for teleconferencing, and spread spectrum communications. Several approaches have been proposed in the literature to detect and estimate the directions-of-arrival (DOA's) of wideband signals. In the most basic approach, the output signal of each sensor is partitioned into nonoverlapping frames or snapshots. In each snapshot, a discrete Fourier transform (DFT) is used to sample the spectrum. For each frequency bin, a narrowband array output signal, with the same DOA's as the wideband signal, is formed. Each one of these narrowband signals is processed individually, and the results are combined to estimate the DOA's [1]. This approach is referred to as the incoherent signal-subspace method (ISM). This method cannot localize completely correlated (coherent) sources; spatial smoothing might be used to handle correlated sources at a cost of reducing array effective aperture [2].

An alternative to the ISM technique is the coherent signalsubspace method (CSM) [3]. In CSM, the correlation matrices at different frequency bins are combined, based on a preestimation of the DOA's, to form a "universal" correlation

Manuscript received August 28, 1995; revised May 15, 1997. The associate editor coordinating the review of this paper and approving it for publication was Dr. Ananthram Swami.

S. Valaee is with the Department of Electrical Engineering, Tarbiat Modares University, Tehran, Iran, and the Department of Electrical Engineering, Sharif University of Technology, Tehran, Iran.

B. Champagne is with INRS-Télécommunications, Verdun, P.Q., Canada H3E 1 H6.

P. Kabal is with the Department of Electrical Engineering, McGill University, Montreal, P.Q., Canada H3A 2A7.

Publisher Item Identifier S 1053-587X(99)03239-0. matrix that is a sufficient statistic for the observation vectors [4]. A high-resolution algorithm, such as MUSIC [5], is applied to this sufficient statistic to estimate the DOA's. In CSM, the combination of the narrowband signals is performed through linear transformation of the observation vectors; this is called focusing. The focusing operator at a given frequency is a matrix that transforms the location matrix of the array at that frequency to the location matrix at the focusing frequency. It has been shown that the CSM algorithm can resolve coherent sources [3]. Furthermore, it has a lower detection threshold as compared with ISM.

An improved version of the CSM uses unitary focusing matrices [6]. This technique is referred to as the rotational signal-subspace (RSS) method. The unitary transformation does not create a focusing loss. The focusing loss is a performance measure proposed in [6] to motivate the use of unitary matrices. In [6], the unitary focusing matrices are determined based on a least-squares (LS) minimization of the norm of the difference between the transformed location matrix at each frequency bin and the focusing location matrix. In [7], it has been shown that if the multiplication of the focusing matrix by its Hermitian transpose is independent of the frequency of operation, the focusing is lossless. A variant of CSM is also defined in [8]. There, the focusing matrix has a diagonal form that leads to computational savings. This method, however, suffers from focusing loss.

The objective of this paper is twofold. First, using the observations of [7], we generalize the technique of [6] to a case in which the focusing subspace is selected as the column span of a transformation of the focusing location matrix. Although we do not expand on it here, this degree of freedom can be exploited to acquire certain properties for the solution to the localization problem. One example can be to optimize the focusing subspace so that the resolution threshold or the bias of the DOA estimation are minimized [9]. We use the leastsquares and the total least-squares (TLS) formulation of the problem and show that under certain constraints, they provide the same solution; this is an extension of the equation solution method discussed in [10]. We then modify the TLS approach (MTLS) to arrive at a new technique for the allocation of the focusing matrices. The technique borrows its name from the TLS solution to linear equations [11] since it permits perturbations in the location matrix at a measured frequency as well as in the location matrix at the focusing frequency. The motivation to use a TLS approach comes from the uncertainty inherent in the measured location matrices due to the error in pre-estimation of the DOA's. Besides sharing 
this uncertainty between the measured location matrix and the focusing matrix, the new technique provides a considerably simpler implementation, as compared with the RSS approach. We also define the focusing gain. We show that the focusing gain of the new technique is larger than the focusing gain of the RSS method. Computer simulation studies show that the MTLS technique outperforms the RSS by reducing the resolution threshold SNR and the bias of DOA estimation.

The paper is organized as follows. A review of the background material on CSM is reported in Section II. The new LSCSM and TLS-CSM algorithms are proposed in Section III. In Section IV, the TLS algorithm is modified and the computational complexity of the new technique is discussed. Section $\mathrm{V}$ deals with the comparison of the new technique to the two-sided correlation transformation (TCT) [12] and the signal-subspace transformation (SST) [7] methods. In Section VI, simulation results are presented.

\section{Coherent Signal-Subspace Method}

Consider $q$ wideband source signals arriving at an array of $p$ sensors from the distinct angles $\theta_{i}, i=1, \cdots, q(q<p)$. The output of each sensor is uniformly sampled and decomposed into nonoverlapping frames or snapshots, each containing $J$ samples. A $J$-point DFT algorithm is used in each snapshot to sample the spectrum of the corresponding signal at a set of discrete frequencies $\omega_{j}, j=1, \cdots, J$. The array output vector for a fixed frequency $\omega_{j}$ is formed by collecting the corresponding samples of all the sensors in a vector. Let the array output vector for frequency $\omega_{j}$ at the $k$ th snapshot be represented by $\boldsymbol{x}_{j, k}$. A model for this vector is given by

$$
\boldsymbol{x}_{j, k}=\boldsymbol{A}_{j}(\boldsymbol{\theta}) \boldsymbol{s}_{j, k}+\boldsymbol{n}_{j, k}
$$

where $\boldsymbol{s}_{j, k}$ and $\boldsymbol{n}_{j, k}$ are the $q \times 1$ signal vector and the $p \times 1$ noise vector, and $\boldsymbol{A}_{j}(\boldsymbol{\theta})=\left[\boldsymbol{a}_{j}\left(\theta_{1}\right) \cdots \boldsymbol{a}_{j}\left(\theta_{q}\right)\right]$ is the $p \times q$ location matrix at frequency $\omega_{j}$. It is assumed that $A_{j}(\boldsymbol{\theta})$ is full rank; at each $\omega_{j}$, the location vectors $\boldsymbol{a}_{j}\left(\theta_{i}\right)$ and $\boldsymbol{a}_{j}\left(\theta_{t}\right)$ are linearly independent for $\theta_{i} \neq \theta_{t}$.

Let the signal and noise be statistically independent from each other with the correlation matrices $S_{j}$ and $\sigma_{j}^{2} N$, respectively. Furthermore, the samples of the signals $\boldsymbol{s}_{j, k}$ and the noise $\boldsymbol{n}_{j, k}$ are independent for different snapshots. For a large time-bandwidth product, samples of the observation vector at different frequency bins are uncorrelated. The correlation matrix of the array at the $j$ th frequency bin can be written as

$$
\boldsymbol{R}_{j}=\boldsymbol{A}_{j}(\boldsymbol{\theta}) \boldsymbol{S}_{j} \boldsymbol{A}_{j}^{H}(\boldsymbol{\theta})+\sigma_{j}^{2} \boldsymbol{N}
$$

where the superscript $H$ represents the Hermitian transposition.

The signal subspace at each frequency bin is defined as the column span of the location matrix at that frequency. For wideband array processing, each frequency bin has a different signal subspace. The CSM technique attempts to transform these subspaces using appropriate matrices in order to align them. This is called focusing, and the transformation matrices are referred to as the focusing matrices. Averaging the transformed correlation matrices gives a "universal" correlation matrix that is used for detection and DOA estimation.
This universal correlation matrix has the characteristics of the correlation matrix of a narrowband configuration with the same DOA's. The universal correlation matrix can be expressed as

$$
\boldsymbol{R}=\boldsymbol{R}_{s}+\boldsymbol{R}_{n}
$$

where $\boldsymbol{R}_{s}$ and $\boldsymbol{R}_{n}$ are the source and the noise universal correlation matrices, respectively, given by

$$
\begin{aligned}
\boldsymbol{R}_{s} & =\sum_{j=1}^{J} \boldsymbol{T}_{j} \boldsymbol{A}_{j}(\boldsymbol{\theta}) \boldsymbol{S}_{j} \boldsymbol{A}_{j}^{H}(\boldsymbol{\theta}) \boldsymbol{T}_{j}^{H} \\
\boldsymbol{R}_{n} & =\sum_{j=1}^{J} \sigma_{j}^{2} \boldsymbol{T}_{j} \boldsymbol{N} \boldsymbol{T}_{j}^{H}
\end{aligned}
$$

where $\boldsymbol{T}_{j}$ is the $j$ th focusing matrix. The DOA's are estimated by performing the eigendecomposition of the matrix pencil $\left(\boldsymbol{R}, \boldsymbol{R}_{n}\right)$ and using the MUSIC algorithm [5].

In practice, the correlation matrix of the array is estimated from the sample correlation matrix

$$
\hat{R}_{j}=\frac{1}{K} \sum_{k=1}^{K} \boldsymbol{x}_{j, k} \boldsymbol{x}_{j, k}^{H} .
$$

The universal sample correlation matrix is then

$$
\hat{\boldsymbol{R}}=\sum_{j=1}^{J} T_{j} \boldsymbol{R}_{j} \boldsymbol{T}_{j}^{H} .
$$

The eigenvalue decomposition is applied to the matrix pencil $\left(\hat{\boldsymbol{R}}, \hat{\boldsymbol{R}}_{n}\right)$, where

$$
\hat{\boldsymbol{R}}_{n}=\sum_{j=1}^{J} \hat{\sigma}_{j}^{2} \boldsymbol{T}_{j} N \boldsymbol{T}_{j}^{H}
$$

and $\hat{\sigma}_{j}^{2}$, which is an estimate of $\sigma_{j}^{2}$, is determined by averaging the $p-q$ smallest eigenvalues of the matrix pencil $\left(\hat{\boldsymbol{R}}_{j}, \boldsymbol{N}\right)$.

The focusing matrices in the original CSM algorithm [3] are the solutions to

$$
A_{0}(\hat{\boldsymbol{\theta}})=\boldsymbol{T}_{j} \boldsymbol{A}_{j}(\hat{\boldsymbol{\theta}}), \quad j=1, \cdots, J
$$

where $\boldsymbol{A}_{0}(\hat{\boldsymbol{\theta}})$ is the focusing location matrix of the array, $\hat{\boldsymbol{\theta}}=\left(\hat{\theta}_{1}, \cdots, \hat{\theta}_{\hat{q}}\right)$ is the vector of focusing DOA's, and $\hat{q}$ is the number of focusing angles. To determine $\hat{q}$ and $\hat{\boldsymbol{\theta}}$, a preprocessing step is required. An ordinary beamformer is applied to estimate the DOA's of the sources. If the angular separation between the sources is smaller than the beamwidth of the beamformer, the spatial spectrum will show a single peak in the vicinity of the actual sources. Thus, closely separated sources may not be resolved at this stage. The locations of the detected peaks serve as the pre-estimated DOA's for focusing. In practice, a few more focusing angles are added in the vicinity of the pre-estimated DOA's [6]. These angles are used to determine the $p \times \hat{q}$ location matrices $\boldsymbol{A}_{0}(\hat{\boldsymbol{\theta}})$ and $A_{j}(\hat{\boldsymbol{\theta}})$. In the original version of the CSM, $A_{0}(\hat{\boldsymbol{\theta}})$ is the array location matrix at the center frequency of the spectrum of the source signal. It has been shown in [9] that a better focusing frequency can be found by minimizing an appropriate criterion. This results in an improved performance in bias and 
resolution threshold SNR. For simplicity of notation, in the sequel, all references to $A_{0}(\hat{\boldsymbol{\theta}})$ and $\boldsymbol{A}_{j}(\hat{\boldsymbol{\theta}})$ will be indicated by $A_{0}$ and $A_{j}$, respectively.

The matrix $\boldsymbol{T}_{j}$ that solves (9) is the focusing matrix of the CSM algorithm at frequency $\omega_{j}$. The signal subspace at the $j$ th frequency bin is the column span of $A_{j}$ and is represented by $\mathcal{S}_{c}\left(A_{j}\right)$. We will refer to $\mathcal{S}_{c}\left(\boldsymbol{A}_{j}\right)$ as the "measured" signalsubspace. Similarly, the focusing signal subspace is the column span of $A_{0}$ and is denoted by $\mathcal{S}_{c}\left(A_{0}\right)$. The multiplication of $\boldsymbol{T}_{j}$ by $\boldsymbol{A}_{j}$ transforms the measured signal subspace at the $j$ th frequency bin into the focusing signal subspace. In general, the linear equation (9) is underdetermined, and its solution $\boldsymbol{T}_{j}$ might be singular and nonunique. However, it is possible to obtain a nonsingular solution to (9) by increasing the number of the focusing angles $\hat{q}$ to the number of sensors $p$ [3].

In [6], a unitary version of the CSM algorithm is introduced, which is based on the following minimization problem:

$$
\begin{array}{ll}
\min _{\boldsymbol{T}_{j}} & \left\|\boldsymbol{A}_{0}-\boldsymbol{T}_{j} \boldsymbol{A}_{j}\right\|^{2}, \quad j=1, \cdots, J \\
\text { s.t. } & \boldsymbol{T}_{j}^{H} \boldsymbol{T}_{j}=\boldsymbol{T}_{j} \boldsymbol{T}_{j}^{H}=\boldsymbol{I}
\end{array}
$$

where $\|\cdot\|$ is the Frobenius norm of a matrix, and $\boldsymbol{I}$ is the identity matrix. It has been shown that the unitary CSM does not create focusing loss. This technique has been termed the rotational signal subspace (RSS) focusing method. The solution to (10) is given by [6], [13]

$$
\boldsymbol{T}_{j}=\boldsymbol{V}_{0} \boldsymbol{V}_{j}^{H}
$$

where $V_{0}$ and $V_{j}$ are the left and the right singular vectors of $A_{0} A_{j}^{H}$.

\section{THE LS-CSM AND TLS-CSM ALGORITHMS}

Lossless focusing is not limited to unitary transformations. In [7], it has been shown that as long as $\boldsymbol{T}_{j} \boldsymbol{T}_{j}^{H}$ is independent of $j$, focusing is lossless. We use this observation to propose a least-squares coherent signal-subspace method (LS-CSM) and a total least-squares coherent signal-subspace method (TLSCSM).

\section{A. $L S-C S M$}

Using [7], the focusing matrices of the coherent signalsubspace method are selected as the solutions to

$$
\begin{array}{cl}
\min _{\boldsymbol{T}_{j}} & \left\|A_{0}-T_{j} A_{j}\right\|^{2}, \quad j=1, \cdots, J \\
\text { s.t. } & \boldsymbol{T}_{j} \boldsymbol{T}_{j}^{H}=\boldsymbol{D}
\end{array}
$$

where $D$ is a Hermitian, positive definite matrix independent of $j$.

Let the square root of $D$ be represented by a Hermitian matrix $C$

$$
C^{2}=D
$$

Let the singular value decomposition of $\boldsymbol{T}_{j}$ be represented by $\boldsymbol{T}_{j}=V_{j} \boldsymbol{\Sigma}_{j} W_{j}^{H}$. Then, $\boldsymbol{T}_{j} \boldsymbol{T}_{j}^{H}=V_{j} \boldsymbol{\Sigma}_{j}^{2} V_{j}^{H}=D=C^{2}$, and $\boldsymbol{C}=\boldsymbol{V}_{j} \boldsymbol{\Sigma}_{j} \boldsymbol{V}_{j}^{H}$. Thus, if $\boldsymbol{U}_{j}=\boldsymbol{V}_{j} \boldsymbol{W}_{j}^{H}$, then

$$
T_{j}=C U_{j}
$$

Note that $\boldsymbol{U}_{j}$ is the closest unitary matrix to $\boldsymbol{T}_{j}$ [13]. The minimization problem (13) can, hence, be written as

$$
\begin{array}{cl}
\min _{\boldsymbol{U}_{j}} & \left\|A_{0}-C U_{j} A_{j}\right\|^{2}, \quad j=1, \cdots, J \\
\text { s.t. } & U_{j} U_{j}^{H}=\boldsymbol{I}
\end{array}
$$

for a fixed $C$ satisfying (15).

The cost function (17) is bounded by

$$
\left\|A_{0}-C U_{j} A_{j}\right\| \leq\|C\| \cdot\left\|C^{-1} A_{0}-U_{j} A_{j}\right\| .
$$

We choose to minimize the upper bound (19) since it is significantly simpler than (17). Furthermore, the upperbound can be viewed as the error criterion of RSS when the focusing location matrix is transformed by $\boldsymbol{C}^{-1}$. In [9], the focusing frequency can be selected based on minimizing an appropriate cost function. A similar technique might be used to determine $C^{-1}$. An alternative is to choose a diagonal $C^{-1}$. Each diagonal element of $\boldsymbol{C}^{-1}$ is considered to be a calibrating factor that is used to correct or modify the response of the corresponding sensor in the focusing subspace. As a third alternative, consider $C^{-1} A_{0}=A_{0} \boldsymbol{A}$, where $\boldsymbol{A}$ is a $\hat{q} \times \hat{q}$ diagonal matrix. In this case, the effect of multiplying $\boldsymbol{C}^{-1}$ by $\boldsymbol{A}_{0}$ is to weight the focusing DOA's. This can be used whenever the uncertainty in the location of the DOA's differs for different angles. Thus, an appropriate $\boldsymbol{C}^{-1}$ can be employed to enhance certain properties of the focusing technique. We do not pursue this discussion further and continue with a general $\boldsymbol{C}$.

As mentioned earlier, we propose using

$$
\begin{array}{cl}
\min _{\boldsymbol{U}_{j}} & \left\|\boldsymbol{C}^{-1} A_{0}-\boldsymbol{U}_{j} \boldsymbol{A}_{j}\right\|^{2}, \quad j=1, \cdots, J \\
\text { s.t. } & \boldsymbol{U}_{j} \boldsymbol{U}_{j}^{H}=\boldsymbol{I}
\end{array}
$$

to determine the focusing matrices of the least-squares coherent signal-subspace method (LS-CSM). The solution to this problem is given by [13]

$$
\boldsymbol{U}_{j}=\boldsymbol{V}_{0} \boldsymbol{V}_{j}^{H}
$$

where $V_{0}$ and $V_{j}$ are now the left and the right singular vectors of $C^{-1} A_{0} A_{j}^{H}$. The corresponding $\boldsymbol{T}_{j}$ is

$$
\boldsymbol{T}_{j}=C \boldsymbol{V}_{0} \boldsymbol{V}_{j}^{H}
$$

Note that for $\boldsymbol{C}=\boldsymbol{I}$, the focusing matrix of the LS-CSM method is identical to the focusing matrix of RSS, which is a special case of the SST technique [7].

An alternative interpretation to the LS-CSM algorithm might be obtained by investigating the error of transformation. Define

$$
\boldsymbol{E}_{0}=U_{j} A_{j}-C^{-1} A_{0}
$$

The LS-CSM problem can be rearranged as

$$
\begin{array}{cl}
\min _{\boldsymbol{T}_{j}} & \left\|\boldsymbol{E}_{0}\right\|^{2} \\
\text { s.t. } & A_{0}+\boldsymbol{C} \boldsymbol{E}_{0}=\boldsymbol{T}_{j} A_{j} \\
& \boldsymbol{T}_{j} \boldsymbol{T}_{j}^{H}=C^{2} .
\end{array}
$$


The constraints in this minimization problem show that the location matrix $A_{0}$ is perturbed such that $A_{j}$ can be transformed onto it using the focusing matrix $\boldsymbol{T}_{j}$. The minimization (25) guarantees that the perturbation matrix $\boldsymbol{E}_{0}$ has the smallest Frobenius norm.

Here, we introduce a lemma that will be used in the following section. This lemma states that the perturbation matrix $\boldsymbol{E}_{0}$ lies in the same subspace as the matrix $\boldsymbol{C}^{-1} \boldsymbol{A}_{0}$.

Lemma 1: Let $\boldsymbol{E}_{0}$ be the solution to (25). The column span of the perturbation matrix $\boldsymbol{E}_{0}$ belongs to the column span of $C^{-1} A_{0}$, i.e.,

$$
\mathcal{S}_{c}\left(\boldsymbol{E}_{0}\right) \subseteq \mathcal{S}_{c}\left(\boldsymbol{C}^{-1} \boldsymbol{A}_{0}\right)
$$

Proof: Using (22), the perturbation matrix $\boldsymbol{E}_{0}$ can be written as

$$
\boldsymbol{E}_{0}=V_{0} V_{j}^{H} A_{j}-C^{-1} A_{0}
$$

Assume that the singular values of $C^{-1} A_{0} A_{j}^{H}$ are arranged in nonincreasing order. Decompose the matrices $V_{0}$ and $V_{j}$ into two submatrices $V_{0}=\left[V_{01} V_{02}\right]$ and $V_{j}=\left[V_{j 1} V_{j 2}\right]$ so that the $p \times \hat{q}$ matrices $V_{01}$ and $V_{j 1}$ correspond to nonzero singular values. Since $\boldsymbol{C}$ is nonsingular and $\boldsymbol{A}_{j}$ is full rank (by assumption), $C^{-1} A_{0} A_{j}^{H}$ spans the same space as $C^{-1} A_{0}$. Thus, $V_{01}$ forms an orthonormal basis for the column span of $\boldsymbol{C}^{-1} \boldsymbol{A}_{0}$. A similar argument indicates that $\boldsymbol{V}_{j 1}$ forms an orthonormal basis for the column span of $\boldsymbol{A}_{j}$. Since $\boldsymbol{V}_{j}$ is a unitary matrix, $\boldsymbol{V}_{j 2}$ will be orthogonal to $\boldsymbol{A}_{j}$. Using these results in (29), we have

$$
\begin{aligned}
\boldsymbol{E}_{0} & =\left[\boldsymbol{V}_{01} \boldsymbol{V}_{02}\right]\left[\begin{array}{c}
\boldsymbol{V}_{j 1}^{H} \\
\boldsymbol{V}_{j 2}^{H}
\end{array}\right] A_{j}-\boldsymbol{C}^{-1} \boldsymbol{A}_{0} \\
& =\boldsymbol{V}_{01} \boldsymbol{V}_{j 1}^{H} \boldsymbol{A}_{j}-\boldsymbol{C}^{-1} \boldsymbol{A}_{0} .
\end{aligned}
$$

Note that $\boldsymbol{V}_{01}$ and $\boldsymbol{C}^{-1} \boldsymbol{A}_{0}$ span the same subspace. Thus, $\boldsymbol{E}_{0}$ is in the column span of $C^{-1} A_{0}$.

\section{B. TLS-CSM}

The total least-squares coherent signal-subspace method (TLS-CSM) is based on perturbing both $A_{0}$ and $\boldsymbol{A}_{j}$. The TLS-CSM transformation matrix is the solution to

$$
\begin{array}{cl}
\min _{\boldsymbol{T}_{j}} & \left(\left\|\boldsymbol{F}_{0}\right\|^{2}+\left\|\boldsymbol{F}_{j}\right\|^{2}\right) \\
\text { s.t. } & A_{0}+\boldsymbol{C} \boldsymbol{F}_{0}=\boldsymbol{T}_{j}\left(\boldsymbol{A}_{j}+\boldsymbol{F}_{j}\right) \\
& \boldsymbol{T}_{j} \boldsymbol{T}_{j}^{H}=\boldsymbol{C}^{2}
\end{array}
$$

where $\boldsymbol{F}_{0}$ and $\boldsymbol{F}_{j}$ are the perturbation matrices.

Lemma 2: Let $\boldsymbol{F}_{0}$ and $\boldsymbol{F}_{j}$ be the solutions to (32). In the TLS approach, the column span of $\boldsymbol{F}_{0}$ and $\boldsymbol{F}_{j}$ belong, respectively, to the column span of $C^{-1} A_{0}$ and $A_{j}$, i.e.,

$$
\begin{aligned}
& \mathcal{S}_{c}\left(\boldsymbol{F}_{0}\right) \subseteq \mathcal{S}_{c}\left(\boldsymbol{C}^{-1} A_{0}\right) \\
& \mathcal{S}_{c}\left(\boldsymbol{F}_{j}\right) \subseteq \mathcal{S}_{c}\left(\boldsymbol{A}_{j}\right)
\end{aligned}
$$

Proof: For any fixed $\boldsymbol{F}_{j}$, using Lemma 1, it is seen that the perturbation matrix $\boldsymbol{F}_{0}$, which has the smallest Frobenius norm, is in the subspace spanned by the column vectors of $\boldsymbol{C}^{-1} \boldsymbol{A}_{0}$. Thus, $\mathcal{S}_{c}\left(\boldsymbol{F}_{0}\right) \subseteq \mathcal{S}_{c}\left(\boldsymbol{C}^{-1} A_{0}\right)$.

Now, fix $\boldsymbol{F}_{0}$, and let $\boldsymbol{T}_{j}=\boldsymbol{C} \boldsymbol{U}_{j}$. The constraint (33) can be written as

$$
\boldsymbol{U}_{j}^{H}\left(\boldsymbol{F}_{0}+\boldsymbol{C}^{-1} A_{0}\right)=A_{j}+\boldsymbol{F}_{j}
$$

Note that the solution to the minimization of $\left\|\boldsymbol{F}_{j}\right\|^{2}$ is obtained from (25) with $\boldsymbol{C}=\boldsymbol{I}$. Using Lemma 1 completes the proof. $\square$

Lemmas 1 and 2 are used to solve the TLS-CSM problem (32), as indicated in the following theorem.

Theorem 1: Let the matrices of the left and the right singular vectors of $C^{-1} A_{0} A_{j}^{H}$ be given by $V_{0}$ and $V_{j}$, respectively. Then, the perturbation matrices

$$
\begin{aligned}
& \boldsymbol{F}_{0}=\frac{1}{2}\left(U_{j} A_{j}-\boldsymbol{C}^{-1} \boldsymbol{A}_{0}\right) \\
& \boldsymbol{F}_{j}=\frac{1}{2}\left(\boldsymbol{U}_{j}^{H} \boldsymbol{C}^{-1} \boldsymbol{A}_{0}-\boldsymbol{A}_{j}\right)
\end{aligned}
$$

solve (32) with the transformation matrix

$$
T_{j}=C U_{j}
$$

where

$$
\boldsymbol{U}_{j}=\boldsymbol{V}_{0} \boldsymbol{V}_{j}^{H}
$$

Proof: See the Appendix.

Note that the focusing matrices for the LS-CSM and TLSCSM techniques are identical. This might be considered to be an extension to the results of [10]. There, it has been shown that if the solution of a set of linear equations is confined to be a unitary matrix, the LS and TLS techniques produce the same results. Here, we have shown that the similarity of the LS and TLS solutions can be extended to the cases where the product of the matrix by its Hermitian transpose is a fixed matrix.

\section{A COMPUTATIONALly EFFICIENT TLS-CSM ALGORITHM}

Lemmas 1 and 2 indicate

$$
\mathcal{S}_{c}\left(A_{0}\right)=\mathcal{S}_{c}\left(\boldsymbol{T}_{j} \boldsymbol{A}_{j}\right)
$$

where $T_{j}$ can be the transformation matrix for either LSCSM or TLS-CSM. Thus, the column span of $A_{j}$ is exactly transformed to the column span of $A_{0}$. This property holds for all signal-subspace transformation techniques used for localization of wideband signals.

The objective of subspace transformation is to transform the array manifold at the measured frequency to the array manifold at the focusing frequency. Since this transformation might not be possible for all angles, if the focusing matrix is confined to a certain class - say, being unitary - the transformation is usually performed in a neighborhood of the estimated DOA's. Thus, if $\boldsymbol{T}_{j} \boldsymbol{A}_{j}$ is close to $\boldsymbol{A}_{0}$, a good estimate of the DOA's might be expected, provided the true DOA's are in the vicinity of the focusing DOA's. Note that the location matrices $A_{j}$ and $A_{0}$ are determined by a preprocessing step (pre-estimation of DOA's) and, hence, contain an error of estimation. Because of uncertainties in $A_{0}$ and $\boldsymbol{A}_{j}$, minimizing the errors $\boldsymbol{F}_{0}$ and $\boldsymbol{F}_{j}$ does not necessarily lead to appropriate results. Here, we relax 
the error constraint in the hope of arriving at a reduction in computation. Based on these arguments, we modify the TLS transformation technique as follows.

If the number of focusing angles is smaller than the number of sensors $(\hat{q}<p)$, the rank of $A_{0}$ and $\boldsymbol{A}_{j}$ is smaller than $p$, and the unitary solution (41) is not unique. Let the matrices $V_{0}$ and $V_{j}$ be decomposed into the forms $V_{0}=\left[V_{01} V_{02}\right]$ and $V_{j}=\left[\boldsymbol{V}_{j 1} V_{j 2}\right]$ so that the $p \times \hat{q}$ matrices $V_{01}$ and $V_{j 1}$ collect the singular vectors corresponding to the nonzero singular values. In such a case, $V_{j 2}^{H} A_{j}=\mathbf{0}$, and we have

$$
T_{j} A_{j}=C\left[V_{01} V_{02}\right]\left[\begin{array}{c}
V_{j 1}^{H} \\
V_{j 2}^{H}
\end{array}\right] A_{j}=C V_{01} V_{j 1}^{H} A_{j}
$$

Thus, for DOA estimation, we could choose $\boldsymbol{U}_{j}=\boldsymbol{V}_{01} \boldsymbol{V}_{j 1}^{H}$. In this case, $U_{j}$ is no longer unitary but has $\hat{q}$ nonzero singular values equal to 1 . Let $\mathcal{T}_{q}(\boldsymbol{C})$ be the set of all $p \times p$ matrices defined as

$$
\begin{gathered}
\mathcal{T}_{q}(\boldsymbol{C})=\left\{\boldsymbol{C M} \mid \boldsymbol{M} \in \mathcal{C}_{p \times p}, \sigma_{i}(\boldsymbol{M})=1, \text { for } i=1, \cdots, q\right. \\
\text { and } \left.\sigma_{i}(\boldsymbol{M})=0, \text { for } i=q+1, \cdots, p\right\}
\end{gathered}
$$

where $\mathcal{C}_{p \times p}$ is the space of $p \times p$ complex matrices, and $\sigma_{i}(\boldsymbol{M})$ is the $i$ th singular value of $\boldsymbol{M}$. Based on (43), the constraint $\boldsymbol{T}_{j} \boldsymbol{T}_{j}^{H}=D=C^{2}$ in (32) might be replaced by $\boldsymbol{T}_{j} \in \mathcal{T}(C)$. Using a technique similar to the proof of Theorem 1, it is possible to show that the solution to (32) is given by

$$
\boldsymbol{T}_{j}=C \boldsymbol{V}_{01} \boldsymbol{V}_{j 1}^{H}
$$

Study of the TLS-CSM technique indicates that the process of focusing can be decomposed into two steps. First, the measured signal-subspace $\mathcal{S}_{c}\left(\boldsymbol{A}_{j}\right)$ is rotated, using $U_{j}$, to the subspace spanned by $C^{-1} A_{0}$. Then, using $C$, the result is transformed into the focusing subspace. We use this view to develop a new technique for wideband signal localization.

Let matrix $\boldsymbol{C}$ be a full-rank transformation that maps $\mathcal{S}_{c}\left(A_{0}\right)$ to itself $\left[\mathcal{S}_{c}\left(\boldsymbol{A}_{0}\right)\right.$ is invariant under $\left.\left.C\right)\right]$; an example would be the identity matrix $(C=I)$, which we use in the sequel. With this assumption, $\left\|A_{0}-C U_{j} A_{j}\right\|=\left\|C^{-1} A_{0}-U_{j} A_{j}\right\|=$ $\left\|A_{0}-\boldsymbol{U}_{j} \boldsymbol{A}_{j}\right\|$. The matrix $\boldsymbol{U}_{j}=\boldsymbol{V}_{01} \boldsymbol{V}_{j 1}^{H}$ transforms $\mathcal{S}_{c}\left(\boldsymbol{A}_{j}\right)$ and overlaps it with $\mathcal{S}_{c}\left(A_{0}\right)$. We choose two orthonormal bases in $\mathcal{S}_{c}\left(A_{0}\right)$ and $\mathcal{S}_{c}\left(\boldsymbol{A}_{j}\right)$ and determine a focusing matrix that transforms the basis of $\mathcal{S}_{c}\left(\boldsymbol{A}_{j}\right)$ onto the basis of $\mathcal{S}_{c}\left(A_{0}\right)$. Let the polar decomposition of the matrices $A_{0}$ and $A_{j}$ be represented by $W_{0} \boldsymbol{Q}_{0}$ and $W_{j} \boldsymbol{Q}_{j}$, respectively, where $\boldsymbol{Q}_{0}$ and $\boldsymbol{Q}_{j}$ are $\hat{q} \times \hat{q}$ Hermitian, positive-definite matrices, and $W_{0}$ and $W_{j}$ are $p \times \hat{q}$ matrices that satisfy $W_{0}^{H} W_{0}=$ $\boldsymbol{I}, \boldsymbol{W}_{j}^{H} \boldsymbol{W}_{j}=\boldsymbol{I}$ [13], [14]. The polar decomposition has the following properties:

1) The matrix $W_{0}$ is the closest matrix (in Frobenius norm) in the set of $p \times \hat{q}$ matrices with orthonormal columns to $A_{0}$. A similar argument holds for $W_{j}$ and $A_{j}$.

2) Since $A_{0}$ is full column rank, the positive-definite matrix $\boldsymbol{Q}_{0}$ is equal to $\left(\boldsymbol{A}_{0}^{H} \boldsymbol{A}_{0}\right)^{1 / 2}$. Similarly, $\boldsymbol{Q}_{j}=\left(\boldsymbol{A}_{j}^{H} \boldsymbol{A}_{j}\right)^{1 / 2}$.

See Fig. 1 for an illustration of the first property.

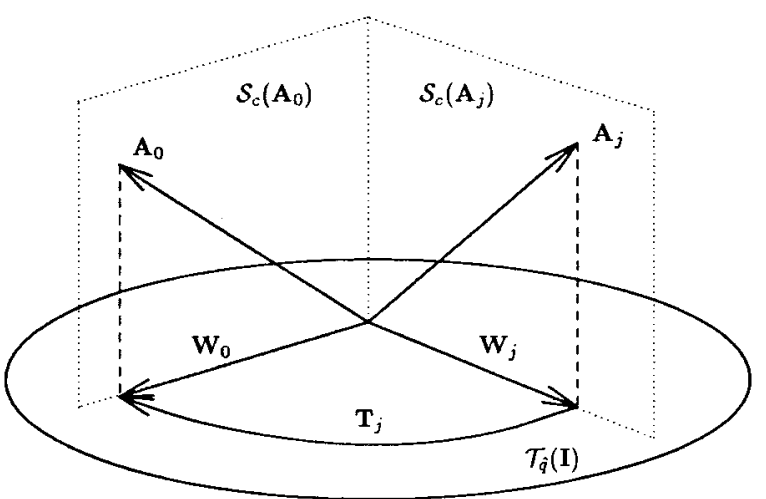

Fig. 1. Geometric interpretation of the MTLS algorithm.

As shown in the figure, the location matrices $\boldsymbol{A}_{0}$ and $\boldsymbol{A}_{j}$ are projected onto $\mathcal{T}_{\hat{q}}(\boldsymbol{I})$. In the set of $p \times \hat{q}$ matrices with orthonormal columns, $W_{0}$ and $W_{j}$ are the closest matrices to $A_{0}$ and $\boldsymbol{A}_{j}$, respectively [13]. A modified total least-squares coherent signal-subspace method (MTLS) can be obtained by choosing the focusing matrix $\boldsymbol{T}_{j}$ as

$$
\boldsymbol{T}_{j}=\boldsymbol{W}_{\mathrm{o}} \boldsymbol{W}_{j}^{H}
$$

The focusing matrix transfers $W_{j}$ to $W_{0}$. In fact, since $A_{0}$ and $A_{j}$ are erroneous, they have been replaced by the approximations $W_{0}$ and $W_{j}$.

The computation of the focusing matrix (46) can further be simplified to

$$
\boldsymbol{T}_{j}=A_{0} Q_{0}^{-1} Q_{j}^{-1} A_{j}^{H}
$$

Using the second property of the polar decomposition, the focusing matrix in the MTLS algorithm is

$$
\boldsymbol{T}_{j}=\boldsymbol{A}_{0}\left(\boldsymbol{A}_{0}^{H} \boldsymbol{A}_{0}\right)^{-(1 / 2)}\left(\boldsymbol{A}_{j}^{H} \boldsymbol{A}_{j}\right)^{-(1 / 2)} \boldsymbol{A}_{j}^{H} .
$$

We will discuss shortly the computation of the focusing matrix $\boldsymbol{T}_{j}$ from (48).

\section{A. Focusing Gain}

The focusing gain is defined as the ratio of SNR at the output of the focusing process to the SNR at the input. The definition of the focusing gain is analogous to the definition of the array gain in beamforming [15]. Using (4) and (5), and assuming $N=I$, the focusing gain is given by

$$
G_{f}=\frac{\operatorname{Tr}\left(\sum_{j=1}^{J} \boldsymbol{T}_{j} \boldsymbol{A}_{j}(\boldsymbol{\theta}) \boldsymbol{S}_{j} \boldsymbol{A}_{j}^{H}(\boldsymbol{\theta}) \boldsymbol{T}_{j}^{H}\right) / \operatorname{Tr}\left(\sum_{j=1}^{J} \sigma_{j}^{2} \boldsymbol{T}_{j} \boldsymbol{T}_{j}^{H}\right)}{\operatorname{Tr}\left(\sum_{j=1}^{J} A_{j}(\boldsymbol{\theta}) \mathcal{S}_{j} A_{j}^{H}(\boldsymbol{\theta})\right) / \operatorname{Tr}\left(\sum_{j=1}^{J} \sigma_{j}^{2} \boldsymbol{I}\right)}
$$

where $\operatorname{Tr}(\cdot)$ is the trace operator. The focusing gain of the RSS technique for the true values of the DOA's is equal to one since $T_{j}$ is unitary - the same as the focusing loss. The focusing gain of MTLS for the true values of the DOA's is 
given by $\left(\boldsymbol{T}_{j}=\boldsymbol{W}_{0} \boldsymbol{W}_{j}^{H}\right)$ in (50), shown at the bottom of the page. For true DOA's, $\boldsymbol{W}_{j}^{H} \boldsymbol{A}_{j}(\boldsymbol{\theta})=\boldsymbol{Q}_{j}$. Using the properties of trace

$$
\begin{gathered}
\operatorname{Tr}\left(\sum_{j=1}^{J} \boldsymbol{W}_{0} \boldsymbol{W}_{j}^{H} \boldsymbol{A}_{j}(\boldsymbol{\theta}) \boldsymbol{S}_{j} \boldsymbol{A}_{j}^{H}(\boldsymbol{\theta}) \boldsymbol{W}_{j} \boldsymbol{W}_{0}^{H}\right) \\
=\operatorname{Tr}\left(\sum_{j=1}^{J} \boldsymbol{Q}_{j} \boldsymbol{S}_{j} \boldsymbol{Q}_{j}\right) \\
\quad=\operatorname{Tr}\left(\sum_{j=1}^{J} \boldsymbol{A}_{j}(\boldsymbol{\theta}) \boldsymbol{S}_{j} \boldsymbol{A}_{j}^{H}(\boldsymbol{\theta})\right) .
\end{gathered}
$$

Now, note that

$$
\operatorname{Tr}\left(\sum_{j=1}^{J} \sigma_{j}^{2} \boldsymbol{W}_{0} \boldsymbol{W}_{j}^{H} \boldsymbol{W}_{j} \boldsymbol{W}_{0}^{H}\right)=q \sum_{j=1}^{J} \sigma_{j}^{2} .
$$

Thus, the focusing gain for the MTLS is

$$
G_{f}^{\mathrm{MTLS}}=\frac{p}{q}>1=G_{f}^{\mathrm{RSS}} .
$$

Intuitively, the MTLS algorithm reduces the noise effect by annihilating the noise components in the noise subspace.

\section{B. The Algorithm}

The MTLS algorithm is summarized as follows.

1) Apply an ordinary beamformer to pre-estimate the DOA's; add a few more angles in the vicinity of the pre-estimated DOA's, and form $\hat{\boldsymbol{\theta}}=\left(\hat{\theta}_{1}, \cdots, \hat{\theta}_{\hat{q}}\right)$.

2) Decompose the output of the sensors into nonoverlapping snapshots, and apply a DFT algorithm in each snapshot to sample the spectrum of data.

3) Form the location matrices $\boldsymbol{A}_{j}, j=1, \cdots, J$, and $\boldsymbol{A}_{0}$.

4) Compute $\left(\boldsymbol{A}_{j}^{H} \boldsymbol{A}_{j}\right)^{-(1 / 2)}$, and $\boldsymbol{W}_{j}=\boldsymbol{A}_{j}\left(\boldsymbol{A}_{j}^{H} \boldsymbol{A}_{j}\right)^{-(1 / 2)}$ for $j=0, \cdots, J$.

5) Determine the universal sample correlation matrix

$$
\hat{\boldsymbol{R}}=W_{0}\left(\sum_{j=1}^{J} W_{j}^{H} \hat{\boldsymbol{R}}_{j} W_{j}\right) W_{0}^{H} .
$$

6) Use AIC or MDL to determine the true number of sources [16].

7) Apply the MUSIC algorithm to estimate the DOAs.

8) To improve the performance, iterate Steps 3)-8).
The bulk of the computational complexity of the new focusing method is due to Step 4 in the above algorithm. To find the square root of the Hermitian, $\hat{q} \times \hat{q}$ matrices $A_{j}^{H} A_{j}, j=$ $0, \cdots, J$, we can use the eigenvalue decomposition or apply an iterative Newton method [14]. It is possible to show that for the Hermitian, positive-definite matrix $B$, if $\boldsymbol{X}_{0}=\boldsymbol{I}$ and

$$
\boldsymbol{X}_{k+1}=\frac{1}{2}\left(\boldsymbol{X}_{k}+B \boldsymbol{X}_{k}^{-H}\right)
$$

then $X_{k} \rightarrow \sqrt{B}$ quadratically, where $\sqrt{B}$ denotes the square root of $\boldsymbol{B}$. To compute $\boldsymbol{A}_{j}\left(\boldsymbol{A}_{j}^{H} \boldsymbol{A}_{j}\right)^{-(1 / 2)}$, we use (55) or the eigenvalue decomposition to find the square root of $A_{j}^{H} A_{j}$ and then multiply $\boldsymbol{A}_{j}$ by the inverse of $\left(A_{j}^{H} \boldsymbol{A}_{j}\right)^{1 / 2}$. Since $A_{j}$ and $A_{0}$ are simply the estimates of the true location matrix, and $\hat{q}$ is usually larger than $q$, a thorough computation of $\left(A_{j}^{H} A_{j}\right)^{-(1 / 2)}$ may not be necessary; a small number of iterations of (55) may suffice.

We compare the MTLS and the RSS techniques based on the number of real flops for computing the focusing matrices. To form the matrices $\boldsymbol{A}_{j}^{H} \boldsymbol{A}_{j}, O\left(p \hat{q}^{2}\right)$ flops are required. Assume that the eigenvalue decomposition is used to determine $\left(\boldsymbol{A}_{j}^{H} \boldsymbol{A}_{j}\right)^{-(1 / 2)}$. An eigenvalue decomposition of $A_{j}^{H} A_{j}$ can be performed in $O\left(\hat{q}^{3}\right)$ flops. Again, $O\left(\hat{q}^{3}\right)$ flops are required to build $\left(\boldsymbol{A}_{j}^{H} \boldsymbol{A}_{j}\right)^{-(1 / 2)}$. The multiplication of $\boldsymbol{A}_{j}$ and $\left(\boldsymbol{A}_{j}^{H} \boldsymbol{A}_{j}\right)^{-(1 / 2)}$ needs $O\left(p \hat{q}^{2}\right)$ flops. Adding these values, the total number of flops in computing each matrix $W_{j}$ in the MTLS algorithm amounts to $O\left(p \hat{q}^{2}\right)$.

In RSS, $O\left(p^{2} \hat{q}\right)$ flops are needed to build $A_{0} A_{j}^{H}$. Then, a $p \times p$ singular value decomposition is performed to acquire the left and right singular vectors of $A_{0} A_{j}^{H}$. This requires $O\left(p^{3}\right)$ flops. The multiplication of the left and the right singular vectors is also performed in $O\left(p^{3}\right)$ flops. Thus, in total, the computational load in RSS is on the order of $O\left(p^{3}\right)$. If $\hat{q} \ll p$, the MTLS algorithm is significantly faster than RSS. Note that since $T_{j}$ is not explicitly formed in the MTLS algorithm, a computation on the order of $O\left(p^{2} \hat{q}\right)$ is saved in the multiplication of the focusing matrices by the sample correlation matrices [see Step 5)].

\section{RELATIONSHIP TO OTHER TECHNIQUES}

Recently, two alternatives to the RSS have been proposed in the literature. In this section, we review the two-sided correlation transformation (TCT) [12] and the signal-subspace transformation (SST) [7] techniques and show their relationship to the MTLS algorithm.

$$
G_{f}^{\mathrm{MTLS}}=\frac{\operatorname{Tr}\left(\sum_{j=1}^{J} \boldsymbol{W}_{0} \boldsymbol{W}_{j}^{H} \boldsymbol{A}_{j}(\boldsymbol{\theta}) \boldsymbol{S}_{j} \boldsymbol{A}_{j}^{H}(\boldsymbol{\theta}) W_{j} W_{0}^{H}\right) / \operatorname{Tr}\left(\sum_{j=1}^{J} \sigma_{j}^{2} \boldsymbol{W}_{0} \boldsymbol{W}_{j}^{H} W_{j} \boldsymbol{W}_{0}^{H}\right)}{\operatorname{Tr}\left(\sum_{j=1}^{J} \boldsymbol{A}_{j}(\boldsymbol{\theta}) \boldsymbol{S}_{j} \boldsymbol{A}_{j}^{H}(\boldsymbol{\theta})\right) / \operatorname{Tr}\left(\sum_{j=1}^{J} \sigma_{j}^{2} \boldsymbol{I}\right)} .
$$




\section{A. Two-Sided Correlation Transformation}

In [12], we have introduced the unitary TCT algorithm for wideband array processing. The focusing transformation matrices in the TCT method are the solutions to

$$
\begin{array}{cl}
\min _{\boldsymbol{U}_{j}} & \left\|\boldsymbol{P}_{0}-U_{j} P_{j} \boldsymbol{U}_{j}^{H}\right\| \quad j=1, \cdots, J \\
\text { s.t. } & \boldsymbol{U}_{j}^{H} \boldsymbol{U}_{j}=\boldsymbol{U}_{j} \boldsymbol{U}_{j}^{H}=\boldsymbol{I}
\end{array}
$$

where $\boldsymbol{P}_{j}$ is the noise-reduced array correlation matrix at the $j$ th frequency bin and is computed from

$$
\boldsymbol{P}_{j}=\hat{\boldsymbol{R}}_{j}-\hat{\sigma}_{j}^{2} \boldsymbol{N}
$$

where $\hat{\boldsymbol{R}}_{j}$ is defined in (6), and $\hat{\sigma}_{j}^{2}$ is an estimate of the noise power at the $j$ th frequency bin; $\boldsymbol{P}_{0}$ is the focusing correlation matrix defined by $\boldsymbol{P}_{0}=A_{0} S_{0} A_{0}^{H}$, where $S_{0}$ is the average of the estimated source correlation matrices

$$
\boldsymbol{S}_{0}=\frac{1}{J} \sum_{j=1}^{J} \hat{\boldsymbol{S}}_{j} .
$$

A solution to (56) is given by

$$
\boldsymbol{U}_{j}=X_{0} \boldsymbol{X}_{j}^{H}
$$

where $\boldsymbol{X}_{0}$ and $\boldsymbol{X}_{j}$ are the eigenvectors of $\boldsymbol{P}_{0}$ and $\boldsymbol{P}_{j}$.

A unitary transformation matrix is chosen in the TCT algorithm to avoid focusing loss. If $X_{j}$ is a matrix with orthonormal columns, $\boldsymbol{U}_{j} \boldsymbol{U}_{j}^{H}$ is equal to $\boldsymbol{X}_{0} \boldsymbol{X}_{0}^{H}$ and independent of $j$, and thus, there is no focusing loss [7]. We might assume that $\boldsymbol{X}_{0}$ and $\boldsymbol{X}_{j}$ are the eigenvectors corresponding to the nonzero eigenvalues of $\boldsymbol{P}_{0}$ and $\boldsymbol{P}_{j}$. In such a case, the focusing matrix is not unitary; however, its nonzero singular values are unity, i.e., $\boldsymbol{U}_{j} \in \mathcal{T}(\boldsymbol{I})$.

\section{B. Signal-Subspace Transformation}

Recently, Doron and Weiss [7] proposed a signal-subspace transformation (SST) by solving

$$
\begin{array}{cl}
\min _{\boldsymbol{T}_{j}} & \left\|A_{0} \boldsymbol{D}_{0} A_{0}^{H}-\boldsymbol{T}_{j} \boldsymbol{A}_{j} \boldsymbol{D}_{j} A_{j}^{H} \boldsymbol{T}_{j}^{H}\right\|^{2} \\
\text { s.t. } & \boldsymbol{T}_{j}^{H} \boldsymbol{T}_{j}=\boldsymbol{T}_{j} \boldsymbol{T}_{j}^{H}=\boldsymbol{I}
\end{array}
$$

where $D_{0}$ and $D_{j}$ are any Hermitian, positive-definite matrices. In their simulations, they chose the identity matrices for $\boldsymbol{D}_{0}$ and $\boldsymbol{D}_{j}$. This results in

$$
\begin{array}{cl}
\min _{\boldsymbol{T}_{j}} & \left\|A_{0} A_{0}^{H}-\boldsymbol{T}_{j} A_{j} \boldsymbol{A}_{j}^{H} \boldsymbol{T}_{j}^{H}\right\|^{2} \\
\text { s.t. } & \boldsymbol{T}_{j}^{H} \boldsymbol{T}_{j}=\boldsymbol{T}_{j} \boldsymbol{T}_{j}^{H}=\boldsymbol{I} .
\end{array}
$$

Theorem 2: The solution to (63) is given by

$$
T_{j}=V_{0} V_{j}^{H} .
$$

where $V_{0}$ and $V_{j}$ are the left singular vectors of $A_{0}$ and $A_{j}$.
Proof: The solution of (63) is given by $\boldsymbol{T}_{j}=\boldsymbol{V}_{0} \boldsymbol{V}_{j}^{H}$, where $V_{0}$ and $V_{j}$ are the eigenvectors of $A_{0} A_{0}^{H}$ and $A_{j} A_{j}^{H}$ [12]. Noting that the eigenvectors of $A_{0} A_{0}^{H}$ and $A_{j} A_{j}^{H}$ are identical to the left singular vectors of $A_{0}$ and $A_{j}$, the proof is complete.

This theorem indicates that unlike MTLS, the SST method only uses the left singular vectors of $\boldsymbol{A}_{0}$ and $\boldsymbol{A}_{j}$. Exploiting the right singular vectors in MTLS results in a better performance. That is because in MTLS, the transformation matrix is formed by multiplying $W_{0}$ and $W_{j}$, which are, respectively, the closest matrices with orthonormal columns to $A_{0}$ and $\boldsymbol{A}_{j}$ (see Fig. 1).

\section{Discussion}

We note that for the RSS, SST, TCT, and MTLS techniques, $\mathcal{S}_{c}\left(\boldsymbol{A}_{0}\right)=\mathcal{S}_{c}\left(\boldsymbol{T}_{j} \boldsymbol{A}_{j}\right)$, where $\boldsymbol{T}_{j}$ is the corresponding focusing matrix. We distinguish these techniques by the method they compose: the unitary focusing matrices. In general, selecting the focusing matrices in any unitary transformation coherent signal-subspace technique comprises two steps. First, some orthonormal bases are found for the measured subspace $\mathcal{S}_{c}\left(\boldsymbol{A}_{j}\right)$ as well as for the focusing subspace $\mathcal{S}_{c}\left(\boldsymbol{A}_{0}\right)$. Then, a unitary matrix (or a matrix with orthonormal columns) is formed, which transforms basis of $\mathcal{S}_{c}\left(\boldsymbol{A}_{j}\right)$ onto the basis of $\mathcal{S}_{c}\left(A_{0}\right)$.

In RSS, the two bases are the left and the right singular vectors of $A_{0} A_{j}^{H}$. The left singular vectors form an orthonormal basis for $\mathcal{S}_{c}\left(A_{0}\right)$, and similarly, the right singular vectors form an orthonormal basis for $\mathcal{S}_{c}\left(\boldsymbol{A}_{j}\right)$. In MTLS, the orthonormal bases $W_{0}$ and $W_{j}$ are the unitary matrices of the polar decomposition of $A_{0}$ and $\boldsymbol{A}_{j}$. The basis $\boldsymbol{W}_{0}$ is the multiplication of the left and the right singular vectors of $A_{0}$ [13]. The same argument holds for $\boldsymbol{W}_{j}$ and $\boldsymbol{A}_{j} . \boldsymbol{W}_{0}$ and $\boldsymbol{W}_{j}$, respectively, are the closest unitary matrices to $A_{0}$ and $A_{j}$ in the Frobenius norm sense [13]. In SST, the orthonormal bases for the measured and the focusing subspaces are the left singular vectors of $A_{0}$ and $A_{j}$. In TCT, the transformation matrix is $\boldsymbol{X}_{0} \boldsymbol{X}_{j}^{H}$, where $\boldsymbol{X}_{0}$ and $\boldsymbol{X}_{j}$ are the eigenvectors of the noise-reduced correlation matrices $\boldsymbol{P}_{0}$ and $\boldsymbol{P}_{j}$. Since $P_{j}$ is measured directly from data by simply subtracting the noise power, it does not contain DOA pre-estimation error. This fact fortifies the TCT with the possibility of producing unbiased estimates of the DOA's in an asymptotic sense; the other techniques discussed here do not have this property.

The computational complexity of SST is on the order of $O\left(p^{2} \hat{q}\right)$, and its performance is almost similar to RSS [7]. TCT has a modest computational complexity relative to RSS, but its capability to generate asymptotically unbiased estimates and its smaller resolution threshold SNR favor its use for wideband array processing. The MTLS algorithm has the smallest computational complexity [on the order of $O\left(p \hat{q}^{2}\right)$ ]. The performance of SST is comparable to RSS (see [7]). TCT outperforms all the others, however, with an increase in the computational complexity [12]. The performance of MTLS is better than that for the RSS as shown in the following section. In Fig. 2, the four techniques are roughly compared in terms of their computational complexity and performance (bias). 


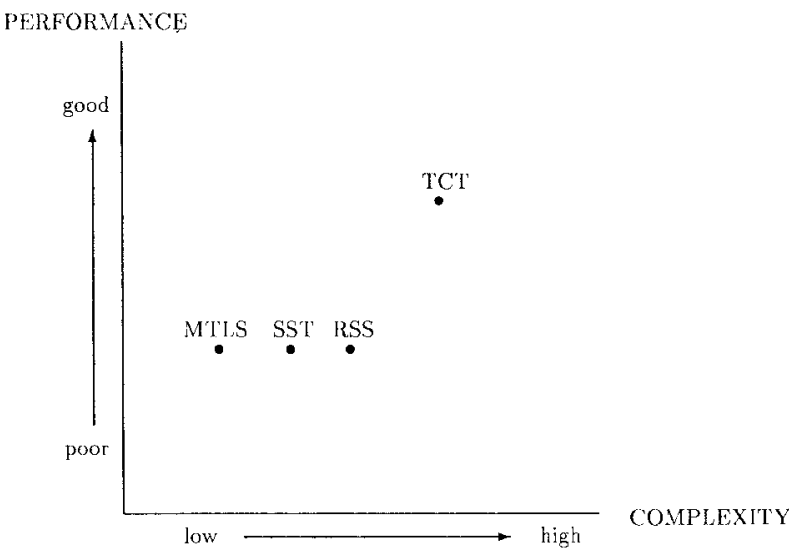

Fig. 2. Comparison of the four techniques: MTLS, SST, RSS, and TCT

TABLE I

Average Bias (IN Degrees) For 100 InDEPENDENT Runs For a SCENARIo with Two Closely Separated Sources at 10 and $14^{\circ}$ Arriving at a Uniform Linear Array of Eight Sensors Using the RSS and MTLS AlgorithmS

\begin{tabular}{cccccc}
\hline & \multicolumn{2}{c}{ RSS } & & \multicolumn{2}{c}{ MTLS } \\
\cline { 2 - 3 } \cline { 5 - 6 } SNR & 10 & 14 & & 10 & 14 \\
\hline 10 & -0.271 & 0.281 & & -0.150 & 0.151 \\
20 & -0.205 & 0.212 & & -0.144 & 0.147 \\
30 & -0.200 & 0.205 & & -0.142 & 0.146 \\
40 & -0.199 & 0.204 & & -0.143 & 0.146 \\
50 & -0.199 & 0.203 & & -0.141 & 0.145 \\
\hline
\end{tabular}

\section{Simulation Results}

In this section, we present the computer simulations that were performed to compare the LS (RSS) and the MTLS approaches to the CSM algorithm with $\boldsymbol{C}=\boldsymbol{I}$. In the first example, we consider a configuration with two equipower coherent wideband sources at the DOA's $10^{\circ}$ and $14^{\circ}$ in the far-field of a uniform linear array of eight sensors. The spacing between each two consecutive sensors is half the wavelength at the center frequency of the spectrum of the wideband signals. The signal of source at $10^{\circ}$ is delayed one sample and introduced as the signal of source at $14^{\circ}$. The sources have a flat complex frequency spectrum over a $40 \%$ relative bandwidth. The center frequency of the spectrum is selected as the focusing frequency. The output of each sensor is decomposed into 100 snapshots of 32 samples each. An FFT algorithm is used in each snapshot to sample the spectrum of signals. A Monte Carlo simulation is performed, and the bias, the standard deviation, and the resolution are averaged over 100 independent runs. At each run, a delayand-sum beamformer is used to estimate the DOA's. Then, two extra angles are added at $1^{\circ}$ from the estimated DOA. This simulation was performed for different SNR's. The focusing matrices in the RSS and MTLS algorithms are computed from (12) and (46), respectively. The average bias and the average standard deviation are reported in Tables I and II. Note that bias of the MTLS is smaller than the bias of the RSS for all SNR's. The difference is significant for small SNR's. The standard deviations of the two techniques are almost identical.

We also compare the peak-to-valley measure of the RSS and the MTLS methods. The peak-to-valley measure is defined as
TABLE II

Average Standard Deviation (In Degrees) For 100 InDEPENDENT Runs For a Scenario with Two Closely SeParated SOURCES AT 10 AND $14^{\circ}$ ARRIVING AT a UNIForm Linear ARray of Eight SENSORs Using the RSS and MTLS Algorithms

\begin{tabular}{cccccc}
\hline & \multicolumn{2}{c}{ RSS } & & \multicolumn{2}{c}{ MTLS } \\
\cline { 2 - 3 } \cline { 5 - 6 } SNR & 10 & 14 & & 10 & 14 \\
\hline 10 & 0.168 & 0.163 & & 0.141 & 0.138 \\
20 & 0.048 & 0.045 & & 0.042 & 0.041 \\
30 & 0.017 & 0.016 & & 0.015 & 0.015 \\
40 & 0.009 & 0.010 & & 0.008 & 0.009 \\
50 & 0.007 & 0.008 & & 0.005 & 0.009 \\
\hline
\end{tabular}

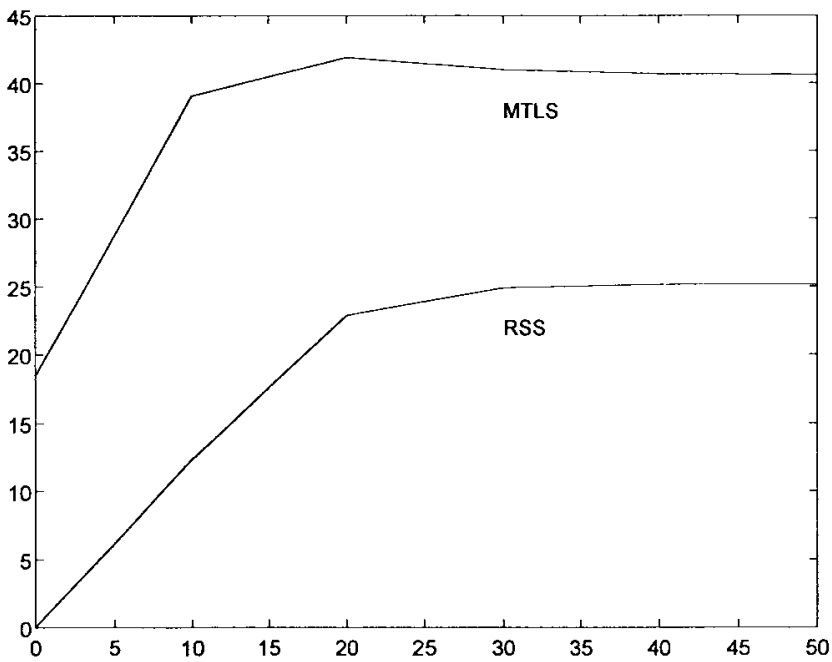

Fig. 3. Peak-to-valley measure of the RSS and MTLS methods averaged over 100 independent runs for a scenario with two closely separated sources at 10 and $14^{\circ}$ arriving at a uniform linear array of eight sensors.

the difference between the average of the spatial spectrum at the peak points in the MUSIC algorithm and the spatial spectrum in the valley [17]. The result is shown in Fig. 3. Note that for small SNR, the MTLS algorithm has a significantly better peak-to-valley measure than the RSS.

To find the probability of resolution threshold, we count the number of times each algorithm resolves the sources. The sources are assumed to be resolved when two peaks in the spatial spectrum of the MUSIC algorithm are in the vicinity (within $1^{\circ}$ ) of the true DOA. Fig. 4 shows the probability of the resolution for the two methods. It is seen that the resolution threshold (defined as the SNR for a fixed probability of resolution) for the MTLS algorithm is smaller than that for the RSS.

Fig. 5 illustrates the focusing gain as a function of the perturbation on the DOA's. As noticed, the maximum focusing gain is obtained for true DOA's.

To study the performance of the MTLS algorithm for multigroup sources, we add two more sources at $33^{\circ}$ and $37^{\circ}$ with the same power and increase the number of sensors to 20 . Table III presents the averaged bias for 100 independent runs. As it is seen, the RSS and the MTLS algorithms both have a small bias. To compare the computational complexity of the two methods, we count the number of flops in the MATLAB software. The location matrices $A_{0}$ and $\boldsymbol{A}_{j}$ are $20 \times 6$ matrices 


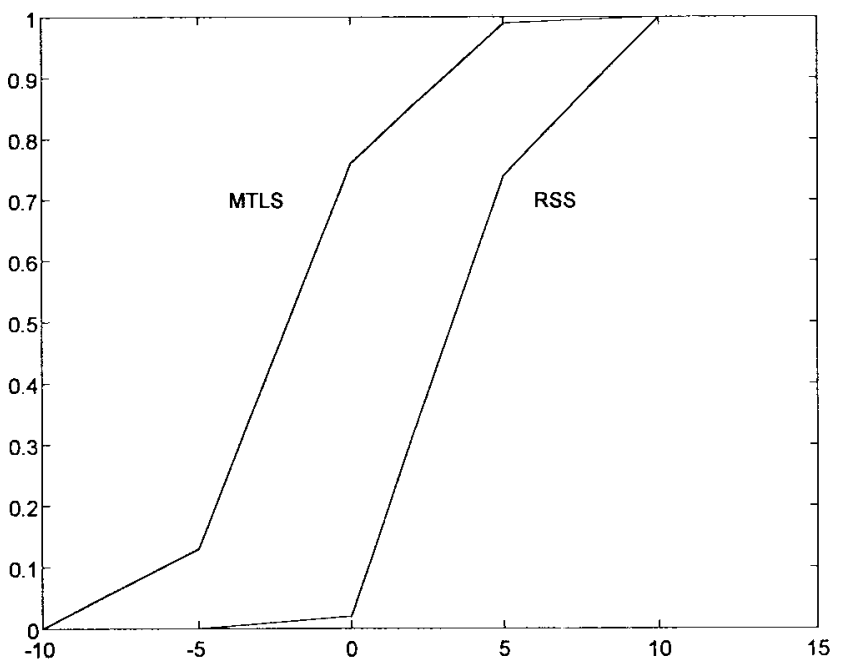

Fig. 4. Probability of resolution for the RSS and MTLS methods for a scenario with two closely separated sources at 10 and $14^{\circ}$ arriving at a uniform linear array of eight sensors.

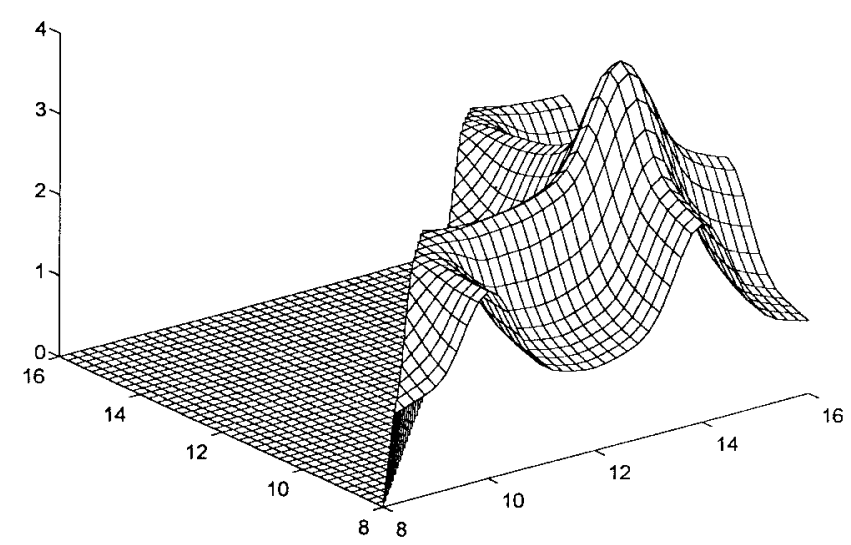

Fig. 5. Focusing gain as a function of perturbation on the DOA's for a scenario with two closely separated sources at 10 and $14^{\circ}$ arriving at a uniform linear array of eight sensors.

TABLE III

Average Bias (IN DEgREES) FOR 100 INDEPENDENT RunS For a Scenario with Four Closely Separated Sources AT $10,14,33$, AND $37^{\circ}$ ARRIVING AT A UNIFORM LINEAR ARRAY OF 20 SENSORS Using THE RSS AND MTLS AlgorithmS

\begin{tabular}{|c|c|c|c|c|c|c|c|c|}
\hline \multirow[b]{2}{*}{ SNR } & \multicolumn{4}{|c|}{ RSS } & \multicolumn{4}{|c|}{ MTLS } \\
\hline & 10 & 14 & 33 & 37 & 10 & 14 & 33 & 37 \\
\hline 0 & -0.03 & 0.04 & -0.03 & 0.03 & -0.03 & 0.03 & -0.01 & 0.04 \\
\hline 10 & -0.03 & 0.04 & -0.03 & 0.03 & -0.04 & 0.03 & -0.01 & 0.04 \\
\hline 20 & -0.03 & 0.04 & -0.03 & 0.03 & -0.04 & 0.03 & -0.01 & 0.04 \\
\hline 30 & -0.03 & 0.04 & -0.03 & 0.03 & -0.03 & 0.03 & -0.01 & 0.04 \\
\hline 40 & -0.03 & 0.04 & -0.03 & 0.03 & -0.04 & 0.03 & -0.01 & 0.04 \\
\hline 50 & -0.03 & 0.04 & -0.03 & 0.03 & -0.03 & 0.03 & -0.01 & 0.04 \\
\hline
\end{tabular}

(corresponding to $p=20$ and $\hat{q}=6$ ). MATLAB uses about 12.9 mega flops to compute all the focusing matrices in the RSS technique. MTLS requires about 1.8 mega flops, which is smaller than that for the RSS by a factor of 7 .

\section{CONCLUSION}

This paper introduces a new focusing technique for the coherent signal-subspace processing method. The least-squares and the total least-squares coherent signal-subspace methods are formulated in a general structure, and it is shown that under certain constraints, the two techniques provide an identical solution. The method can be viewed as an extension to nonunitary focusing techniques in the CSM algorithm. We then use this formulation to derive a new focusing algorithm. The focusing matrices in the new algorithm are determined based on a modification to the total least-squares method (MTLS). We show that the new technique can increase the focusing gain achieved by a unitary coherent signal-subspace method. The computer simulations verify that the new technique has a better than or equal to performance than RSS and a smaller computational cost. In particular, it is shown that the new method has a smaller resolution signal-to-noise ratio and a smaller bias than RSS. The computational complexity of MTLS is smaller than that for the RSS.

\section{APPENDIX \\ PROOF OF THEOREM 1}

We use the Lagrange multipliers method to solve (32). The Lagrangian is defined as

$$
\begin{aligned}
\ell= & \left\|\boldsymbol{F}_{0}\right\|^{2}+\left\|\boldsymbol{F}_{j}\right\|^{2}+\mathbf{1}_{\hat{q}}^{T}\left[\alpha \odot \left(\boldsymbol{A}_{0}+\boldsymbol{C} \boldsymbol{F}_{0}\right.\right. \\
& \left.\left.-\boldsymbol{T}_{j}\left(A_{j}+\boldsymbol{F}_{j}\right)\right)\right] \mathbf{1}_{\hat{q}}+\mathbf{1}_{\hat{q}}^{T}\left[\beta \odot\left(\boldsymbol{T}_{j} \boldsymbol{T}_{j}^{H}-\boldsymbol{C}^{2}\right)\right] \mathbf{1}_{\hat{q}}
\end{aligned}
$$

where $\alpha$ and $\eta$ are the matrices of Lagrange multipliers, $\mathbf{1}_{\hat{q}}$ is a $\hat{q} \times 1$ vector with all components equal to 1 , the superscript $T$ denotes transposition, and $\odot$ is the Hadamard product defined by

$$
[\boldsymbol{A} \odot \boldsymbol{B}]_{i j}=a_{i j} b_{i j}
$$

for two matrices $A=\left[a_{i j}\right]$ and $B=\left[b_{i j}\right]$. The derivative of $\ell$ with respect to a complex variable $z$ is defined as

$$
\frac{\partial \ell}{\partial z}=\frac{1}{2}\left(\frac{\partial \ell}{\partial z_{R}}-j \frac{\partial \ell}{\partial z_{I}}\right)
$$

where $z_{R}$ and $z_{I}$ are the real and imaginary parts of the complex variable $z$. We also define the derivative with respect to a matrix $\boldsymbol{B}=\left[b_{i j}\right]$ as

$$
\frac{\partial \ell}{\partial B}=\left[\frac{\partial \ell}{\partial b_{i j}}\right] .
$$

Using these definitions, we have

$$
\begin{aligned}
& {\left[\frac{\partial \ell}{\partial \boldsymbol{F}_{0}}\right]^{*}=\boldsymbol{F}_{0}+C \alpha^{*}=0} \\
& {\left[\frac{\partial \ell}{\partial \boldsymbol{F}_{j}}\right]^{*}=\boldsymbol{F}_{j}-T_{j}^{H} \alpha^{*}=0}
\end{aligned}
$$

where []$^{*}$ denotes the complex conjugation. These equations are solved along with

$$
\begin{aligned}
A_{0}+C \boldsymbol{F}_{0} & =T_{j}\left(A_{j}+\boldsymbol{F}_{j}\right) \\
T_{j} \boldsymbol{T}_{j}^{H} & =C^{2}
\end{aligned}
$$

to get $\boldsymbol{F}_{0}$ and $\boldsymbol{F}_{j}$. (70) and (71) give

$$
\boldsymbol{F}_{j}=-T_{j}^{H} \boldsymbol{C}^{-1} \boldsymbol{F}_{0}
$$


From (73)

$$
T_{j}=C U_{j}
$$

where $U_{j} U_{j}^{H}=I$. Substituting these matrices in (72) gives

$$
\boldsymbol{F}_{0}=\frac{1}{2}\left(\boldsymbol{U}_{j} \boldsymbol{A}_{j}-\boldsymbol{C}^{-1} A_{0}\right) \text {. }
$$

Use $\boldsymbol{F}_{0}$ in (74) to get

$$
\boldsymbol{F}_{j}=\frac{1}{2}\left(\boldsymbol{U}_{j}^{H} \boldsymbol{C}^{-1} \boldsymbol{A}_{0}-\boldsymbol{A}_{j}\right) .
$$

The error is given by

$$
\left\|\boldsymbol{F}_{0}\right\|^{2}+\left\|\boldsymbol{F}_{j}\right\|^{2}=\frac{1}{2}\left\|\boldsymbol{U}_{j} \boldsymbol{A}_{j}-\boldsymbol{C}^{-1} A_{0}\right\|^{2} .
$$

The matrix $\boldsymbol{U}_{j}$ should be selected to minimize the error. Let the left and the right singular vectors of $C^{-1} A_{0} A_{j}^{H}$ are given by $V_{0}$ and $V_{j}$, respectively. The unitary matrix that minimizes (78) is then given by [13]

$$
U_{j}=V_{0} V_{j}^{H} \text {. }
$$

\section{REFERENCES}

[1] M. Wax, T. Shan, and T. Kailath, "Spatio-temporal spectral analysis by eigenstructure methods," IEEE Trans. Acoust., Speech, Signal Processing, vol. ASSP-32, pp. 817-827, Aug. 1984.

[2] T. J. Shan, M. Wax, and T. Kailath, "On spatial smoothing for directionof-arrival estimation of coherent signals," IEEE Trans. Acoust., Speech, Signal Processing, vol. ASSP-33, pp. 806-811, Aug. 1985.

[3] H. Wang, and M. Kaveh, "Coherent signal-subspace processing for the detection and estimation of angles of arrival of multiple wide-band sources," IEEE Trans. Acoust., Speech, Signal Processing, vol. ASSP-33, pp. 823-831, Aug. 1985.

[4] H. Hung and M. Kaveh, "On the statistical sufficiency of the coherently averaged covariance matrix for the estimation of the parameters of wideband sources," in Proc. IEEE Int. Conf. Acoust., Speech, Signal Process., 1987.

[5] R. O. Schmidt, "Multiple emitter location and signal parameter estimation," IEEE Trans. Antennas Propagat., vol. AP-34, pp. 276-280, Mar. 1986.

[6] H. Hung and M. Kaveh, "Focusing matrices for coherent signal-subspace processing," IEEE Trans. Acoust., Speech, Signal Processing, vol. 36, pp. 1272-1281, Aug. 1988.

[7] M. A. Doron and A. J. Weiss, "On focusing matrices for wideband array processing,” IEEE Trans. Signal Processing, vol. 40, pp. 1295-1302, June 1992.

[8] J. Krolik, Advances in Spectrum Analysis and Array Processing, S. Haykin, Ed. Englewood Cliffs, NJ: Prentice-Hall, vol. 2, ch. 6, 1991.

[9] S. Valaee and P. Kabal, "Selection of the focusing frequency in wideband array processing," in Proc. 16th Biennial Symp. Commun., Kingston, Ont., Canada, May 1992, pp. 410-414.

[10] K. S. Arun, " A unitarily constrained total least squares problem in signal processing," SIAM J. Matrix Anal. Appl., vol. 13, pp. 729-745, July 1992.

[11] G. H. Golub and C. F. Van Loan, "An analysis of the total least squares problem," SIAM J. Numer. Anal., vol. 17, pp. 883-893, Dec. 1980.

[12] S. Valaee and P. Kabal, "Wideband array processing using a two-sided correlation transformation," IEEE Trans. Signal Processing, vol. 43, pp. 160-172, Jan. 1995.

[13] R. A. Horn and C. A. Johnson, Matrix Analysis. Cambridge, U.K.: Cambridge Univ. Press, 1985
[14] N. J. Higham, "Computing the polar decomposition-with applications," SIAM J. Sci. Stat. Comput., vol. 7, pp. 1160-1174, Oct. 1986.

[15] D. H. Johnson and D. E. Dudgeon, Array Signal Processing: Concepts and Techniques. Englewood Cliffs, NJ: Prentice-Hall, 1993.

[16] M. Wax, "Detection of coherent and noncoherent signals via the stochastic signal model," in Proc. IEEE Int. Conf. Acoust., Speech, Signal Process., May 1991, pp. 3541-3544.

[17] C. Zhou, F. Habre, and D. L. Jaggard, "A resolution measure for the MUSIC algorithm and its application to plane wave arrivals contaminated by coherent interference," IEEE Trans. Signal Processing, vol. 39, pp. 454-463, Feb. 1991

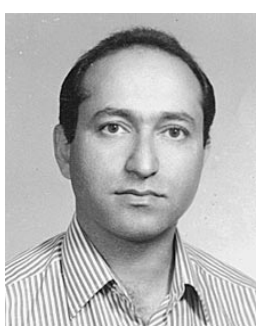

Shahrokh Valaee was born in Tabriz, Iran. He received the B.Sc. and M.Sc. degrees from Tehran University, Tehran, Iran, and the Ph.D. degree from McGill University, Montreal, P.Q., Canada, all in electrical engineering.

From August 1994 to September 1995, he was a Research Associate at INRS Telecommunications, University of Quebec, Montreal. Since 1996, he has been an Assistant Professor with the Department of Electrical Engineering, Tarbiat Modares University, Tehran, and an Adjunct Professor with the Department of Electrical Engineering, Sharif University of Technology, Tehran. He is also a consultant to the Iranian Telecommunications Research Center, working in the field of ATM networks. His major focus of research is in the area of detection and parameter estimation as applied to array processing, harmonic resolution, and spectrum estimation and, recently, congestion control in ATM networks.

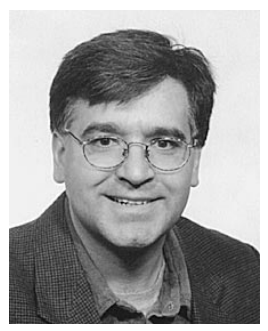

Benoit Champagne (M'89) was born in Joliette, P.Q., Canada, on January 13, 1961. He received the B.Ing. degree in engineering physics from the Ecolé Polytechnique of Montréal, Montréal, P.Q., in 1983, the M.Sc. degree in physics from the University of Montréal in 1985, and the Ph.D. degree in electrical engineering from the University of Toronto, Toronto, Ont., Canada, in 1990.

In June 1990, he joined INRS-Télécommunications, Université du Québec, Verdun, P.Q., where he is presently an Associate Professor. Since September 1994, he has also been an Adjunct Professor with the Department of Electrical Engineering, McGill University, Montréal. His current research interests are in the areas of statistical signal processing and adaptive filtering, including subspace tracking, array processing and beamforming, subband adaptive filtering, and echo cancellation.

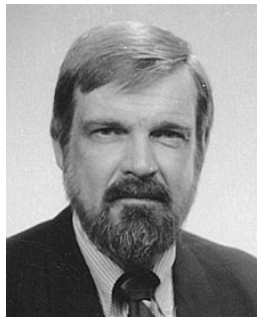

Peter Kabal (M'75) received the B.A.Sc., M.A.Sc., and $\mathrm{Ph} . \mathrm{D}$. degrees in electrical engineering from the University of Toronto, Toronto, Ont., Canada.

$\mathrm{He}$ is a Professor with the Department of Electrical and Computer Engineering, McGill University, Montreal, P.Q., Canada, and is also a Visiting Professor with INRS-Telecommunications, Université du Québec, Verdun, P.Q. His current research interests focus on digital signal processing as applied to speech and audio coding, adaptive filtering, and data transmission. 